\title{
A CHARACTERISTIC NUMBER FOR LINKS OF SURFACE SINGULARITIES
}

\author{
JONATHAN WAHL
}

\section{INTRODUCTION}

Milnor and Thurston [MT] define a characteristic number of a closed orientable 3-manifold $M$ to be a real-valued topological invariant $\varphi(M)$ such that: if $\varphi(M)$ is defined, and $\widetilde{M}$ is a $k$-sheeted covering of $M$, then $\varphi(\widetilde{M})$ is defined and equal to $k \varphi(M)$. This multiplicative property is clear for the Euler characteristic, but of course this is 0 for a 3-manifold. If $M$ has an appropriate Riemannian metric (e.g., hyperbolic, or with all sectional curvatures +1 ), then the volume is a characteristic number. Milnor and Thurston introduce several characteristic numbers; one is defined for all 3-manifolds, is positive on a fixed hyperbolic manifold, and has the additional property that if $M \rightarrow N$ is a general map of degree $k$, then $\varphi(M) \geq|k| \varphi(N)$. Another characteristic number is the simplicial volume introduced by Gromov [G].

In this paper, we introduce a characteristic number for each link of a complex surface singularity. It is a nonnegative rational number, is 0 only in wellunderstood cases, is computable from any resolution dual graph (=plumbing diagram), and has in addition the submultiplicative property above for degree $k$ maps arising from morphisms of the singularities themselves. This is the only characteristic number for links which we know of that can be computed from the graph. We call this invariant $-P \cdot P$, since it is the negative of the self-intersection number of a (rational) cycle on a complex surface (a resolution of the singularity).

The definition arose from our work on the generalized Miyaoka inequality for normal surfaces [W3] (this term appeared in the inequality). Another ingredient is the notion of Zariski decomposition of a line bundle or divisor on a surface, especially on a resolution of a surface singularity, as in Sakai [S].

Let $(X, o)$ be the germ of a normal complex surface singularity (necessarily isolated) with $X$ contractible, and $\partial X=M$ the link of $X . M$ is a closed, connected, orientable 3-manifold. Let $(\tilde{X}, E) \rightarrow(X, o)$ be a good resolution; hence, the inverse image $E=\bigcup E_{i}$ of $o$ is the union of nonsingular curves, intersecting transversally, no three through a point. The resolution dual graph

Received by the editors July $20,1989$.

1980 Mathematics Subject Classification (1985 Revision). Primary 14J17; Secondary 57N10.

This research was partially supported by the National Science Foundation. 
is the information of the genera of the $E_{i}$ and the (negative-definite) intersection matrix $\left(E_{i} \cdot E_{j}\right)$. An important theorem of W. Neumann [N1] asserts that the fundamental group of the link determines the resolution dual graph (up to blowing up and down), except for links of cyclic quotient singularities and cusps (in which cases our invariant will be 0 anyway). Now, the aforementioned work of Sakai implies that any line bundle $\mathscr{L}$ on $\widetilde{X}$ admits a Zariski decomposition $\mathscr{L}=P+N$, where $P$ and $N$ are certain uniquely determined rational combinations of the $E_{i}$ 's. Denote by $K$ the canonical line bundle (or a canonical divisor) on $\widetilde{X}$. In (2.8) and (2.9) below, we prove the following

Theorem. Suppose $(\widetilde{X}, E) \rightarrow(X, o)$ is a good resolution of a normal surface singularity. Consider the Zariski decomposition $K+E=P+N$. Then

(a) $-P \cdot P \in \mathbb{Q}$ is a nonnegative characteristic number for the link $M$ of $X$, is independent of $\widetilde{X}$, and depends only on $\pi_{1}(M)$.

(b) $-P \cdot P=0$ if and only if $\pi_{1}(M)$ is finite or solvable if and only if $(X, o)$ is a log-canonical singularity.

(c) If $(Y, o) \rightarrow(X, o)$ is a finite map of degree $d$, then

$$
-P_{Y} \cdot P_{Y} \geq d\left(-P_{X} \cdot P_{X}\right) \text {. }
$$

(d) $\operatorname{dim} H^{0}(\tilde{X}-E, \mathscr{O}(n(K+E))) / H^{0}(\tilde{X}, \mathscr{O}(n(K+E)))=n^{2} / 2(-P \cdot P)+$ $O(n)$.

To show $-P \cdot P$ is characteristic, note that the isomorphism $\pi_{1}(M) \approx$ $\pi_{1}(X-\{0\})$ means that finite covering spaces of $M$ correspond to finite analytic maps $(Y, o) \rightarrow(X, O)$ which are unramified off $\{0\}$; it therefore suffices to check multiplicativity under such finite maps.

As motivation for the main theorem, consider characteristic numbers for smooth projective surfaces. One has of course the Chern numbers, $c_{1}^{2}=K \cdot K$ and $c_{2}$. If one wishes this number to be a birational invariant as well, there is $\chi\left(\mathscr{O}_{X}\right)=1 / 12 \cdot\left(c_{1}^{2}+c_{2}\right)$. But for nonruled surfaces, there is one more such invariant: $K \cdot K$ of the minimal model of $X$ ! Using the Zariski decomposition $K=P+N$ on $X$ (which exists when $X$ is nonruled), this invariant equals $P \cdot P$, and also satisfies $H^{0}(n K)=n^{2} \cdot / 2 \cdot(P \cdot P)+O(n)$.

Corollary. The image of a log-canonical surface singularity under a finite map is also log-canonical.

Corollary. A finite self-map $(X, o) \rightarrow(X, o)$ of a non-log-canonical singularity is an automorphism.

The log-canonical surface singularities (see (2.4)) were classified by Kawamata $[\mathrm{K}]$; their links are especially simple from the topological point of view, and can also be listed from our approach. We know of no other way to prove either Corollary except as a consequence of (b) and (c) of the main theorem. One can use the same point-of-view to prove that a finite rational self-map of a surface of general type is birational (and hence a biregular automorphism). 
The invariant $-P \cdot P$ has been considered in another guise by $\mathrm{K}$. Watanabe [Wt]. He defines the $n$th plurigenus of $(X, o)$ to be

$$
\begin{aligned}
\delta_{n} & =\operatorname{dim} H^{0}(X-\{o\}, \mathscr{O}(n K)) / L^{2 / n}(X-\{0\}) \\
& =\operatorname{dim} H^{0}(\tilde{X}-E, \mathscr{O}(n(K+E))) / H^{0}(\widetilde{X}, \mathscr{O}(n K+(n-1) E)),
\end{aligned}
$$

and proves

$$
\delta=\lim \sup \delta_{n} / n^{2}<\infty .
$$

Now, $\operatorname{dim} H^{0}\left(E, \mathscr{O}_{E}\left(n\left(K_{\tilde{X}}+E\right)\right)\right)$ grows at most linearly in $n$, and is a bound for the difference between $\delta_{n}$ and the dimension of (d) of the Theorem. One concludes that $-P \cdot P=2 \delta$, allowing one to either deduce or use some of the two-dimensional results of [Wt].

We expect that $-P \cdot P$ has many of the nice properties of the invariant $K \cdot K$ studied by $\mathrm{H}$. Laufer (e.g., [L]). It should be semicontinuous, and its constancy in a family should imply simultaneous resolution of the "log-minimal" model of $X$; the latter is obtained by collapsing some cyclic quotient configurations on the minimal good resolution, so that the $\mathbb{Q}$-divisor $\dot{K}+E$ becomes relatively ample (cf. $[\mathrm{K}])$.

Aside from (d) above, giving the invariant as an asymptotic growth term, we have no other interpretations in general of $-P \cdot P$; however, we prove $((3.2)$ and (3.4)) the

Theorem. Let $(X, O)$ be quasi-homogeneous and let $M$ be the Seifert manifold which is the link. Suppose $X$ is not log-canonical. Then there is a natural metric on $M$ so that the volume is $4 \pi^{2}(-P \cdot P)$.

In the last theorem, the universal covering of $M$ is $\operatorname{PSL}(2, \mathbb{R})^{\sim}$, which has a natural metric (see [N2, p. 251]).

Links of singularities are very special 3-manifolds; they can be spliced together using Seifert manifolds, and have no hyperbolic pieces. Still, the last theorem raises the question of whether there is some metric on a general link $M$ whose volume is essentially $-P \cdot P$. In this regard, we ask the

Conjecture. Let $(X, o)$ be a Gorenstein singularity, not log-canonical (i.e., not a rational double point, a simple elliptic, or a cusp). Then $-P \cdot P \geq 1 / 42$, with equality only for the $(2,3,7)$-triangle singularity or its equisingular deformation.

For a hypersurface singularity, this conjecture would follow from the semicontinuity of $-P \cdot P$, since one can check the singularities of low codimension. We prove the conjecture in the quasi-homogeneous case in $\S 3$, motivated by the boundedness below of volumes of certain quotients of the Poincare disk.

It is also natural to ask whether a similar invariant may be defined for graph three-manifolds which are defined by a nondegenerate (as opposed to negativedefinite) intersection matrix.

In the first section of the paper, we review the Zariski decomposition of a general line bundle $\mathscr{L}$ on $\widetilde{X}$, and interpret $-P \cdot P$ in terms of asymptotic growth 
of some cohomology groups (Theorem 1.6). (This part may be skipped completely if one is not interested in the asymptotic part (d) of the main Theorem above.) In the second section, we discuss $K+E$ and its Zariski decomposition, and describe the log-canonical singularities; we then prove (Theorems 2.8 and 2.9 ) the main results quoted above. Finally, the third section considers the case of quasi-homogeneous singularities.

\section{ZARISKI DECOMPOSITION AND RIEMANN-ROCH}

(1.1) Let $(\widetilde{X}, E) \rightarrow(X, o)$ be a resolution of a complex normal surface singularity. We shall assume for convenience that the resolution is good $(E=$ $E_{1} \cup \cdots \cup E_{s}$ is the transversal union of smooth curves, no three through a point), $X$ is Stein and contractible, and $\partial X=M$ is a closed oriented manifold (the link of $X)$. Since $\left(E_{i} \cdot E_{j}\right)$ is negative definite, there is an adjoint homomorphism

$$
\operatorname{Pic} \tilde{X} \rightarrow \bigoplus \mathbb{Q} \cdot E_{i} \equiv \mathbb{E}_{\mathbb{Q}},
$$

associating to $\mathscr{L}$ the rational Cartier divisor $\sum a_{i} E_{i}$ satisfying

$$
\mathscr{L} \cdot E_{j}=\left(\sum a_{i} E_{i}\right) \cdot E_{j}, \quad \text { all } j .
$$

We shall denote the image of $\mathscr{L}, \mathscr{M}, \ldots$, by $L, M, \ldots$, while $K$ will denote both the canonical line bundle and its image in $\mathbb{E}_{\mathbb{Q}}$.

Proposition 1.2 (Sakai [S, p. 408]). Let $L \in \mathbb{E}_{\mathbb{Q}}$ be a rational Cartier divisor. Then there exists a unique Zariski-decomposition $L=P+N$ in $\mathbb{E}_{\mathbb{Q}}$, where

(a) $P$ is nef, i.e., $P \cdot E_{i} \geq 0$, all $i$.

(b) $N$ is effective, i.e., $N=\sum a_{i} E_{i}$, with all $a_{i} \geq 0$.

(c) $P \cdot N=0$, i.e., $P \cdot E_{i}=0$, all $E_{i} \subset \operatorname{Supp} N$.

Proof. We prove existence only, following [S]. If $L \cdot E_{i} \geq 0$, all $i$, we are done. If not, let $\mathscr{A}_{1}=\left\{j \mid L \cdot E_{j}<0\right\}$. Let $N_{1}=\sum b_{j} E_{j} \quad\left(j \in \mathscr{A}_{1}\right)$ be defined by

$$
N_{1} \cdot E_{j}=L \cdot E_{j}, \quad \text { all } j \in \mathscr{A}_{1}
$$

since these quantities are $<0, N_{1}$ is effective. Letting $P_{1}=L-N_{1}$, we have $P_{1} \cdot E_{j}=0, j \in \mathscr{A}_{1}$, and $P_{1} \cdot N_{1}=0$; so if $P_{1} \cdot E_{i} \geq 0$, all $i$, then $L=P_{1}+N_{1}$ is the desired decomposition. Otherwise, let $\mathscr{A}_{2}^{\prime}=\left\{j \mid P_{1} \cdot E_{j}<0\right\}, \mathscr{A}_{2}=\mathscr{A}_{1} \cup \mathscr{A}_{2}^{\prime}$, and define $N_{2}=\sum c_{k} E_{k}\left(k \in \mathscr{A}_{2}\right)$ so that $P_{1} \cdot E_{k}=N_{2} \cdot E_{k}$, all $k \in \mathscr{A}_{2}$. Thus, $N_{2} \cdot E_{k} \leq 0$, all $k \in \mathscr{A}_{2}$, so $N_{2}$ is effective, and $P_{2}=P_{1}-N_{2}$ is orthogonal to the curves corresponding to the (larger) index set $\mathscr{A}_{2}$. Eventually, some $P_{r}$ is nef and orthogonal to all the curves in the union of the supports of the effective Q-divisors $N_{1}, \ldots, N_{r}$. Letting $P=P_{r}, N=N_{1}+\cdots+N_{r}$ gives the Zariski decomposition.

(1.3) Zariski decomposition for a line bundle $\mathscr{L}$ may be understood as follows: There is an integer $r>0$ and an effective integral divisor $Z$ so that $\mathscr{M}=\mathscr{L}^{\otimes r}(-Z)$ has degree $\geq 0$ when restricted to any $E_{i}$, and degree 0 if $E_{i} \subset|Z|$. Numerically, one has a Zariski decomposition $L=(1 / r) M+(1 / r) Z$. 
(1.4) Zariski decomposition is preserved under pull-back, in the following sense. Suppose $\tilde{f}:(\tilde{Y}, F) \rightarrow(\widetilde{X}, E)$ is a generically finite and proper surjective map of resolutions covering $(Y, o) \rightarrow(X, o)(\tilde{f}$ is finite off $F)$. Then $\tilde{f}^{*}$ induces a map $\tilde{f}^{*}: \mathbb{E}_{\mathbb{Q}} \rightarrow \mathbb{F}_{\mathbb{Q}}$ which preserves both effective and nef divisors, and which multiplies intersection numbers by $d=\operatorname{deg}(f)$. So if $L=P+N$ is a Zariski decomposition, so is $\tilde{f}^{*} L=\tilde{f}^{*} P+\tilde{f}^{*} N$.

(1.5) The rest of this section is devoted to a sharp form of Riemann-Roch for powers of a line bundle $\mathscr{L}$. Let $U=\widetilde{X}-E=X-\{o\}, h^{i}(\mathscr{L})=$ $\operatorname{dim} H^{i}(\tilde{X}, \mathscr{L})$, and $\mathscr{L}^{n}=\mathscr{L}^{\otimes n}$. Then according to [Mo, Theorem 1.4], we have

$\operatorname{dim} H^{0}\left(U, \mathscr{L}^{n}\right) / H^{0}\left(\tilde{X}, \mathscr{L}^{n}\right)+h^{1}\left(\mathscr{L}^{n}\right)=n^{2} / 2(-L \cdot L)+n / 2(L \cdot K)+b(n)$, where $b(n)$ is a bounded function of $n$.

Theorem 1.6. Let $\mathscr{L} \in \operatorname{Pic}(\tilde{X})$ be a line bundle and $L=P+N$ the Zariski decomposition. Then for every $n \geq 1$,

$$
\operatorname{dim} H^{0}\left(U, \mathscr{L}^{n}\right) / H^{0}\left(\tilde{X}, \mathscr{L}^{n}\right)=n^{2} / 2(-P \cdot P)+n / 2(P \cdot K)+b_{1}(n),
$$

$$
h^{1}\left(\mathscr{L}^{n}\right)=n^{2} / 2(-N \cdot N)+n / 2(N \cdot K)+b_{2}(n) \text {, }
$$

where $b_{1}(n)$ and $b_{2}(n)$ are bounded functions of $n$.

Lemma 1.7 (cf. [Mo, 1.3.2.2]). Let $(\tilde{X}, E) \rightarrow(X, o)$ be a resolution, $E=$ $E_{1} \cup \cdots \cup E_{s}$, and $n_{1}, \ldots, n_{s}$ given integers. Then there exists a constant $C$ so that $\mathscr{L} \cdot E_{i} \geq n_{i}$, all $i \Rightarrow h^{1}(\mathscr{L}) \leq C$.

Proof of Lemma 1.7. It is well known that if a line bundle $\mathscr{M}$ satisfies $\mathscr{M} \cdot E_{i} \leq$ 0 , all $i$, then $H_{E}^{1}(\mathscr{M})=0$, or dually $H^{1}\left(\mathscr{M}^{-1} \otimes K\right)=0$ (e.g., [W1]). Let $Z$ be an (effective) divisor so that

$$
Z \cdot E_{i} \leq \min \left(0, n_{i}-K \cdot E_{i}\right), \quad \text { all } i .
$$

Then $\mathscr{L} \cdot E_{i} \geq n_{i}$ implies $K \otimes \mathscr{L}^{-1}(Z) \cdot E_{i} \leq 0$, so the vanishing above gives $H^{1}(\mathscr{L}(-Z))=0$. Thus, $h^{1}(\mathscr{L})=h^{1}\left(\mathscr{L} \otimes \mathscr{O}_{Z}\right)$. For an exceptional $E_{i}$ in the support of an effective integral divisor $Y$, one has an exact sequence

$$
0 \rightarrow \mathscr{O}_{E_{i}}\left(-\left(Y-E_{i}\right)\right) \rightarrow \mathscr{O}_{Y} \rightarrow \mathscr{O}_{Y-E_{i}} \rightarrow 0
$$

so that

$$
h^{1}\left(\mathscr{L} \otimes \mathscr{O}_{Y}\right) \leq h^{1}\left(\mathscr{L} \otimes \mathscr{O}_{Y-E_{i}}\right)+h^{1}\left(\mathscr{L}\left(-\left(Y-E_{i}\right)\right) \otimes \mathscr{O}_{E_{i}}\right) .
$$

Given $Y$, one can always find an $E_{i}$ in its support with $Y \cdot E_{i} \leq 0$. For this curve, one has

$$
\mathscr{L}\left(-\left(Y-E_{i}\right)\right) \cdot E_{i} \geq n_{i}-E_{i} \cdot E_{i}
$$

by Riemann-Roch, $h^{1}\left(\mathscr{L}\left(-\left(Y-E_{i}\right)\right) \otimes \mathscr{O}_{E_{i}}\right)$ has dimension which is bounded above independent of $\mathscr{L}$. By induction, we deduce $h^{1}\left(\mathscr{L} \otimes \mathscr{O}_{Z}\right)$ is bounded 
by an expression depending only on $Z$ and the $n_{i}$ 's. This completes the proof of Lemma 1.7.

Proof of Theorem 1.6. As in (1.3), write $\mathscr{L}^{r}=\mathscr{M}(Z) . \quad Z$ is an effective integral divisor, $\mathscr{M} \cdot E_{i} \geq 0$, and $\mathscr{M} \cdot E_{i}=0$ if $E_{i} \subset \operatorname{Supp}(Z)$. The Zariski decomposition of $\mathscr{L}$ is $P+N$, where $P=(1 / r) M$ and $N=(1 / r) Z$.

To study $\mathscr{L}^{n}$, we write $n=m r+t, 0 \leq t<r$. There are exact sequences

$$
0 \rightarrow \mathscr{L}^{m r+t}(-m Z) \rightarrow \mathscr{L}^{m r+t} \rightarrow \mathscr{L}^{m r+t} \otimes \mathscr{O}_{m Z} \rightarrow 0 .
$$

We have

$$
\begin{aligned}
\mathscr{L}^{m r+t}(-m Z) \cdot E_{i} & =(m r+t) L \cdot E_{i}-m Z \cdot E_{i} \\
& =(m r+t) P \cdot E_{i}+t N \cdot E_{i} \geq t N \cdot E_{i}, \quad \text { all } i .
\end{aligned}
$$

Thus, by Lemma $1.7, h^{1}\left(\mathscr{L}^{m r+t}(-m Z)\right)$ takes on finitely many values, independent of $m$.

Next, let $F=\operatorname{Supp}(Z)$. Then

$$
h^{0}\left(\mathscr{L}^{m r+t} \otimes \mathscr{O}_{m Z}\right)=h^{0}\left(\mathscr{L}^{m r+t}(-m Z) \otimes \mathscr{O}_{m Z}(m Z)\right) \leq h_{F}^{1}\left(\mathscr{L}^{m r+t}(-m Z)\right) \text {, }
$$

where the last inequality is, e.g., by [W1, 2.2]. By duality, and viewing $F$ as the exceptional divisor of the resolution $(\widetilde{Y}, F)$ of several other singularities, one has

$$
h_{F}^{1}\left(\mathscr{L}^{m r+t}(-m Z)\right)=h^{1}\left(K_{\tilde{Y}}(m Z) \otimes \mathscr{L}^{-(m r+t)}\right) .
$$

If $E_{i} \subset F=\operatorname{Supp}(Z)=\operatorname{Supp}(N)$, then $P \cdot E_{i}=0$, so $L \cdot E_{i}=N \cdot E_{i}$, and the last line bundle restricted to $E_{i}$ has degree

$$
K_{\widetilde{Y}} \cdot E_{i}+m r N \cdot E_{i}-(m r+t) N \cdot E_{i}=K_{\widetilde{Y}} \cdot E_{i}-t N \cdot E_{i} .
$$

Again by Lemma 1.7, the $h^{1}$ of (1.6.4) takes on finitely many values, independent of $m$; this is thus true of $h^{0}\left(\mathscr{L}^{m r+t} \otimes \mathscr{O}_{m Z}\right)$. But the usual version of Riemann-Roch and the adjunction formula gives

$$
\chi\left(\mathscr{L}^{m r+t} \otimes \mathscr{O}_{m Z}\right)=-\frac{1}{2} m Z \cdot(m Z+K)+(m r+t) L \cdot m Z .
$$

Simplifying, we find that

$$
\begin{aligned}
h^{1}\left(\mathscr{L}^{m r+t} \otimes \mathscr{O}_{m Z}\right) & =-N \cdot N m r(m r+2 t) / 2+\frac{1}{2} m r N \cdot K+b(n) \\
& =-(m r+t)^{2} / 2 N \cdot N+\frac{1}{2}(m r+t) N \cdot K+b_{1}(n),
\end{aligned}
$$

where the $b$ 's are bounded functions of $n=m r+t$. Combining with (1.6.3) and the remark thereafter, we have proved (1.6.2). Then (1.6.1) follows either by reexamination of (1.6.3), or most simply comparing (1.6.2) with (1.5.1) and noting that $L \cdot L=P \cdot P+N \cdot N$ and $L \cdot K=P \cdot K+N \cdot K$.

\section{ZARISKI DECOMPOSITION OF $K+E$}

(2.1) Let $(X, o)$ be a normal surface singularity, with link $M .(X, o)$ is a quotient singularity if it is analytically equivalent to $\left(\mathbb{C}^{2} / G, o\right)$, where 
$G \subset \mathrm{GL}(2, \mathbb{C})$ is a finite subgroup; alternatively, $\pi_{1}(M)$ is finite. $(X, o)$ is simple elliptic if there is a resolution with $E$ a smooth elliptic curve; or $M$ is a circle bundle over a torus. $(X, o)$ is a cusp singularity if there is a resolution for which $E$ is a cycle of smooth rational curves, each intersecting exactly two others; or $M$ is a 2-torus bundle over the circle. A simple elliptic singularity may have a cyclic group of order $2,3,4$, or 6 acting freely off the origin; and some cusps have a similar action by $\mathbb{Z} / 2$. The quotients by these actions together with the first three types are called log-canonical singularities (see 2.4 below for an explanation). According to $[\mathrm{Wg}]$, these are exactly the singularities for which $\pi_{1}(M)$ is solvable or finite.

(2.2) Let $(\widetilde{X}, E) \rightarrow(X, o)$ be the minimal good resolution. For the remainder of this section, we will consider the Zariski decomposition of $K_{\tilde{X}}+E$ :

$$
K+E=P+N .
$$

It is well known that if $X$ is a quotient singularity, then $P=0$ (e.g., $[\mathrm{K}]$ ); if $X$ is a simple elliptic or cusp singularity, then $K+E=0$. In all other cases, $N$ is computed as follows:

A string $S$ in $E$ is a chain of smooth rational curves $E_{1}, \ldots, E_{n}$ so that $E_{i} \cdot E_{i+1}=1 \quad(i=1, \ldots, n-1)$, and these account for all intersections of the $E_{j}$ in $E$, except that $E_{n}$ intersects exactly one other curve. Let $a_{i}=$ $-E_{i} \cdot E_{i}$. Then $S$ can be blown down to a cyclic quotient singularity of order $\Delta$, computable from the $a_{i}$ 's via continued fraction expansion. Let $D=\sum b_{i} E_{i}$ $\left(b_{i} \in \mathbb{Q}, 1 \leq i \leq n\right)$ be the cycle such that $D \cdot E_{1}=-1, D \cdot E_{i}=0(i>1)$; thus, $b_{n}=1 / \Delta, b_{n-1}=a_{n} / \Delta$, etc., and the $b_{i}$ are positive.

Proposition 2.3. Suppose $X$ is not a quotient, simple elliptic, or cusp singularity. Let $N=\sum D_{j}$ be the sum over each maximal string $S_{j}$ in $E$ of the corresponding cycle $D_{j}$. Then $N$ is the negative part of the Zariski decomposition of $K+E$, with $P=K+E-N$.

Proof. First, $N$ is effective. For any exceptional curve $E_{i}$, let $g_{i}=\operatorname{genus}\left(E_{i}\right)$ and $t_{i}=$ number of intersections of $E_{i}$ with other curves $E_{j}$. Then

$$
P \cdot E_{i}=\left(K+E-\sum D_{j}\right) \cdot E_{i}=2 g_{i}-2+t_{i}-\left(\sum D_{j}\right) \cdot E_{i} .
$$

If $E_{i}$ is in a string but is not on an end, then $g_{i}=0, t_{i}=2$, and $E_{i}$ dots to 0 with its $D$-cycle by definition; therefore, $P \cdot E_{i}=0$. Similarly, an end of a string dots to 0 with $P$. Thus, $P \cdot N=0$. It remains to check that $P$ is nef.

So, assume $E_{i}$ is not in a string, but intersects $r \geq 0$ strings, with discriminants $\Delta_{1}, \ldots, \Delta_{r} ;$ then

$$
P \cdot E_{i}=2 g_{i}-2+t_{i}-\sum\left(1 / \Delta_{j}\right) .
$$

Each $\Delta_{j} \geq 2$ (by the minimality of the good resolution), and $r \leq t_{i}$; one deduces easily that $P \cdot E_{i} \geq 0$ unless $g_{i}=0, t_{i}=r=3$, and $\sum\left(1 / \Delta_{j}\right)>1$; but this is the excluded quotient singularity case. This completes the proof. 
Remark 2.4. A singularity is defined to be log-canonical if $P=0$, i.e., $K+$ $E=N$. (This is independent of the resolution, as follows by (2.8) below.) Excluding the quotient, simple elliptic, and cusp singularities, one may examine Proposition 2.3 to find the remaining log-canonical singularities. One first finds all $g_{i}=0, t_{i} \leq 4$; and if some $t_{i}=4$, the resolution dual graph has shape

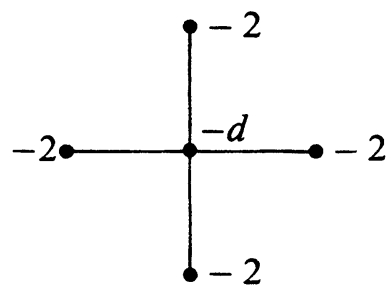

One easily deduces the other remaining types (cf. $[\mathrm{K}]$ ).

Question 2.5. A resolution has $N_{\tilde{X}}=0$ exactly when there are no rational strings on the graph. Is every $(X, o)$ the Galois quotient $(Y, o) \rightarrow(X, o)$, unramified off $o$, of a singularity possessing a resolution $\tilde{Y}$ with $N_{\widetilde{Y}}=0$ ? A result of Pinkham [P] (quoted in the proof of 3.1 below) gives an affirmative answer in the quasi-homogeneous case, and we have checked it for several other examples.

Example 2.6. Consider the $D_{p, q, r}$-singularity $(2 \leq p \leq q \leq r, 1 / p+1 / q+$ $1 / r<1)$, with resolution dual graph

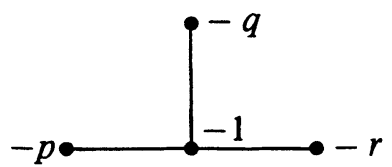

Call the exceptional curves $C_{1}, C_{2}, C_{3}$ (of self-intersections $-p,-q,-r$ ), and $C$. Then $K+E=-C$, and

$$
N=(1 / p) C_{1}+(1 / q) C_{2}+(1 / r) C_{3} .
$$

In particular, we compute that with $P=K+E-N$,

$$
-P \cdot P=-(1-1 / p-1 / q-1 / r) \text {. }
$$

(2.7) Let $(\tilde{X}, E) \rightarrow(X, o)$ be any good resolution, $\pi:\left(X^{\prime}, F\right) \rightarrow(\tilde{X}, E)$ the blow-up of a point $q$ of $E$, and $F_{1}=\pi^{-1}(q)$. One has

$$
K_{X^{\prime}}+F=\pi^{*}\left(K_{\tilde{X}}+E\right)+\delta F_{1},
$$

where $\delta=1$ if $q$ is a smooth point of $E$, and $\delta=0$ if $q$ is a double point. One therefore has

$$
K_{X^{\prime}}+F=\pi^{*}\left(P_{\tilde{X}}\right)+\left(\pi^{*} N_{\tilde{X}}+\delta F_{1}\right)
$$

and this is easily seen to be the Zariski decomposition. In particular, $P_{X^{\prime}}=$ $\pi^{*} P_{\widetilde{X}}$. It follows that $-P_{X^{\prime}} \cdot P_{X^{\prime}}=-P_{\widetilde{X}} \cdot P_{\widetilde{X}}$, a nonnegative rational number; we therefore have a resolution invariant, which we denote $-P_{X} \cdot P_{X}$. In fact, 
we have

Theorem 2.8. Let $(\tilde{X}, E) \rightarrow(X, o)$ be a good resolution of a normal surface singularity, and consider the Zariski decomposition $K_{\tilde{X}}+E=P_{\widetilde{X}}+N_{\widetilde{X}}$. Then (2.8.1) $-P_{\widetilde{X}} \cdot P_{\widetilde{X}} \equiv-P_{X} \cdot P_{X}$ is a nonnegative rational number, independent of the resolution.

(2.8.2) $-P_{X} \cdot P_{X}$ is 0 iff $(X, o)$ is log-canonical.

(2.8.3) If $f:(Y, o) \rightarrow(X, o)$ is finite surjective and unramified off $f^{-1}(o)=$ $\{0\}$, then

$$
-P_{Y} \cdot P_{Y}=(\operatorname{deg} f) \cdot\left(-P_{X} \cdot P_{X}\right) .
$$

(2.8.4) $\operatorname{dim} H^{0}(\tilde{X}-E, \mathscr{O}(n(K+E))) / H^{0}(\tilde{X}, \mathscr{O}(n(K+E)))=n^{2} / 2(-P \cdot P)+$ $O(n)$.

Proof. For the first two statements, it remains only to add that for any exceptional $\mathbb{Q}$-divisor $P$, one has $-P \cdot P \geq 0$, with equality iff $P=0$. (2.8.4) is an application of Theorem 1.6. For (2.8.3), let $(\tilde{X}, E) \rightarrow(X, o)$ be a good resolution, and $Y^{\prime} \rightarrow \tilde{X}$ the normalization of $\tilde{X}$ in the function field of $(Y, o)$. Then this last map is unramified off $E$, and $Y^{\prime}$ has only cyclic quotient singularities. Let $\tilde{Y} \rightarrow Y^{\prime}$ be the minimal resolution of the singularities of $Y^{\prime}$, and $(\widetilde{Y}, F) \rightarrow(Y, o)$ the corresponding resolution of $(Y, o)$. For the induced map $\tilde{f}:(\tilde{Y}, F) \rightarrow(\tilde{X}, E)$, a simple argument in local coordinates gives

$$
\tilde{f}^{*} \Omega_{\widetilde{X}}^{1}(\log E) \cong \Omega_{\widetilde{Y}}^{1}(\log F) ;
$$

taking determinants,

$$
\tilde{f}^{*}\left(K_{\tilde{X}}+E\right) \cong K_{\tilde{Y}}+F .
$$

It follows that one has the Zariski decomposition

$$
K_{\tilde{Y}}+F=\tilde{f}^{*}\left(P_{\widetilde{X}}\right)+\tilde{f}^{*}\left(N_{\tilde{X}}\right) \text {. }
$$

Therefore, $P_{\widetilde{Y}}=\tilde{f}^{*} P_{\widetilde{X}}$. As intersection numbers multiply by degree under pull-back, (2.8.3) is verified.

Theorem 2.9. Let $f:(Y, o) \rightarrow(X, o)$ be a finite surjective morphism between normal surface singularities, of degree $d$. Then

$$
-P_{Y} \cdot P_{Y} \geq d\left(-P_{X} \cdot P_{X}\right) \text {. }
$$

Proof. By blowing-up sufficiently a resolution of $X$, one may obtain a generically finite map $\tilde{f}:(\tilde{Y}, F) \rightarrow(\widetilde{X}, E)$ between resolutions of the two spaces, inducing $f$; we may also assume $\tilde{f}$ has strong normal crossings divisors for both branch locus and ramification locus. $\tilde{f}$ factors as the composition of $h: Y^{\prime} \rightarrow \widetilde{X}$ (the normalization of $\tilde{X}$ in the fraction field of $\tilde{Y}$ ) and a resolution of cyclic quotient singularities $g: \tilde{Y} \rightarrow Y^{\prime}$. We write the reduced branch locus on $\tilde{X}$ as $E+R$ (where $R$ has no proper components), and the reduced ramification locus on $\tilde{Y}$ as $F+R^{\prime}$. Then (as mentioned in the proof of 2.8) a local argument gives

$$
\tilde{f}^{*}\left(K_{\tilde{X}}+E+R\right)=K_{\widetilde{Y}}+F+R^{\prime}
$$


Therefore,

$$
\tilde{f}^{*}\left(K_{\tilde{X}}+E\right)\left(\tilde{f}^{*}(R)-R^{\prime}\right)=K_{\widetilde{Y}}+F
$$

We note that as divisors on $\tilde{Y}, \tilde{f}^{*}(R)-R^{\prime}=R^{\prime \prime}+Z$, where $R^{\prime \prime}$ is effective and supported on $R^{\prime}$, and $Z$ is an effective exceptional divisor. Further, $Z$ is supported in the exceptional locus of $g: \tilde{Y} \rightarrow Y^{\prime}$, since $h^{*}(R)$ contains no proper components. We consider the inclusion of three line bundles on $\tilde{Y}$ :

$$
\mathscr{L}=\tilde{f}^{*}\left(K_{\tilde{X}}+E\right) \subset \mathscr{L}^{\prime}=\mathscr{L}(Z) \subset \mathscr{L}^{\prime \prime}=\mathscr{L}^{\prime}\left(R^{\prime \prime}\right)=K_{\tilde{Y}}+F .
$$

We will prove the result by comparing the growth rate of several functions of the form

$$
\chi^{\prime}(\mathscr{M})=\operatorname{dim} H^{0}(V, \mathscr{M}) / H^{0}(\tilde{Y}, \mathscr{M}), \quad \text { where } V=\tilde{Y}-F=Y-\{o\},
$$

and $\mathscr{M}$ is a line bundle on $\tilde{Y}$. First, since the positive part of $\tilde{f}^{*}\left(K_{\tilde{X}}+E\right)$ is $\tilde{f}^{*}\left(P_{\tilde{X}}\right)$, we have by $(1.6 .1)$

$$
\begin{aligned}
\chi^{\prime}\left(\mathscr{L}^{n}\right) & =\chi^{\prime}\left(\tilde{f}^{*}\left(K_{\tilde{X}}+E\right)^{n}\right)=n^{2} / 2 \cdot\left(-\tilde{f}^{*} P_{\widetilde{X}} \cdot \tilde{f}^{*} P_{\widetilde{X}}\right)+O(n) \\
& =n^{2} / 2 \cdot(\operatorname{deg} \tilde{f})\left(-P_{\widetilde{X}} \cdot P_{\widetilde{X}}\right)+O(n) \\
& =n^{2} / 2 \cdot(\operatorname{deg} f)\left(-P_{X} \cdot P_{X}\right)+O(n) .
\end{aligned}
$$

Next, again by (1.6.1) we have

$$
\begin{aligned}
\chi^{\prime}\left(\mathscr{L}^{\prime \prime n}\right) & =\chi^{\prime}\left(\left(K_{\widetilde{Y}}+F\right)^{n}\right)=n^{2} / 2 \cdot\left(-P_{\widetilde{Y}} \cdot P_{\widetilde{Y}}\right)+O(n) \\
& =n^{2} / 2 \cdot\left(-P_{Y} \cdot P_{Y}\right)+O(n) .
\end{aligned}
$$

We can therefore complete the proof of the theorem if we show

$$
\chi^{\prime}\left(\mathscr{L}^{n}\right)=\chi^{\prime}\left(\mathscr{L}^{\prime n}\right) \text { and } \chi^{\prime}\left(\mathscr{L}^{\prime n}\right) \leq \chi^{\prime}\left(\mathscr{L}^{\prime \prime n}\right) .
$$

Comparing terms of highest degree will give the desired inequality.

For the first equality, note first that $\mathscr{L}$ is trivial in a neighborhood of $Z$, since $\mathscr{L}$ is the pull-back of a line bundle on $Y^{\prime}$ while $Z$ is exceptional for $\widetilde{Y} \rightarrow Y^{\prime}$. This gives the exact sequence

$$
0 \rightarrow \mathscr{L}^{n} \rightarrow \mathscr{L}^{\prime n} \rightarrow \mathscr{O}_{n Z}(n Z) \rightarrow 0
$$

Since $H^{0}$ of the third term vanishes by a standard argument (e.g., [W1]), we have $H^{0}(\widetilde{Y},-)$ of the first two terms are equal. Since the two line bundles agree on $V=\widetilde{Y}-F$, we deduce the equality in (2.9.2).

For the inequality in (2.9.2), it is clear from the definitions that it suffices to prove

$$
H^{0}\left(\tilde{Y}-F, \mathscr{L}^{\prime n}\right) \cap H^{0}\left(\tilde{Y}, \mathscr{L}^{\prime \prime n}\right)=H^{0}\left(\tilde{Y}, \mathscr{L}^{\prime n}\right) .
$$

However, this equality is true by local considerations simply because $\mathscr{L}^{\prime \prime}$ differs from $\mathscr{L}^{\prime}$ by an effective divisor containing no components of $F$; if a section of $\mathscr{L}^{\prime n}$ over $\widetilde{Y}-F$ acquired a pole over $F$, it could not extend as a holomorphic section of $\mathscr{L}^{\prime \prime n}$ (which only allows new poles over $R^{\prime \prime}$ ). 


\section{3. $-P \cdot P$ FOR SEIFERT MANIFOLDS}

(3.1) Let $(X, o)$ be a quasi-homogeneous singularity. Thus, $X$ admits a representation by an affine variety with a good $\mathbb{C}^{*}$-action, or equivalently is defined by weighted homogeneous polynomials. Then the link $M$ of $X$ is a Seifert manifold (see [N2] for more discussion). Suppose $X$ is not a cyclic quotient singularity; then there is a good resolution for which the graph consists of one smooth central curve $C$, of genus $g$ and self-intersection $-b$, and $r$ strings of rational curves emanating from $C$, whose self-intersections are described by the continued fraction expansions of $\alpha_{i} / \beta_{i} \quad\left(0<\beta_{i}<\alpha_{i}\right.$, $\left.\left(\beta_{i}, \alpha_{i}\right)=1, i=1, \ldots, r\right)$ :

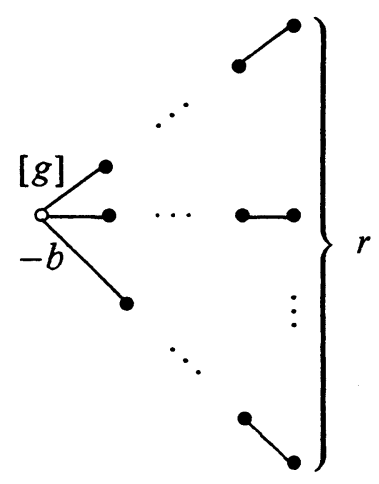

Specifically, the self-intersections of the $i$ th string are $-a_{1}, \ldots,-a_{k}$, reading out from the middle, where these numbers are given by the continued fraction expansion $\alpha_{i} / \beta_{i}=a_{1}-1 \sqrt{a_{2}}-1\left\lceil\cdots-1 \sqrt{a_{k}}\right.$. The set $\left\{g ; b,\left(\alpha_{1}, \beta_{1}\right), \ldots\right.$, $\left.\left(\alpha_{r}, \beta_{r}\right)\right\}$ are the Seifert invariants of the link $M$. (Unfortunately, the sign conventions for these invariants are not uniform-the definition is slightly different in [N2].)

Theorem 3.2 (cf. [Wt, Corollary 2.25]). Let $(X, o)$ be a quasi-homogeneous singularity which is not log-canonical, and with resolution data $\left\{g ; b,\left(\alpha_{1}, \beta_{1}\right)\right.$, $\left.\ldots,\left(\alpha_{r}, \beta_{r}\right)\right\}$. Then one has

$$
-P_{X} \cdot P_{X}=\frac{\left(2 g-2+r-\sum\left(1 / \alpha_{i}\right)\right)^{2}}{b-\sum\left(\beta_{i} / \alpha_{i}\right)} .
$$

Proof. By [P, Theorem 1.1], there is a Galois covering $f:(Y, o) \rightarrow(X, o)$, unramified off $o$, for which $Y$ is a cone; thus, $Y$ has a resolution $\widetilde{Y}$ containing a single smooth exceptional curve $C^{\prime}$, of genus $g^{\prime}$ and self-intersection $-b^{\prime}$. It is easy to see that $K_{\widetilde{Y}}+C^{\prime}=\left(2-2 g^{\prime}\right) / b^{\prime} \cdot C^{\prime}=P_{\widetilde{Y}}$, whence

$$
-P_{Y} \cdot P_{Y}=\left(2 g^{\prime}-2\right)^{2} / b^{\prime} \text {. }
$$

Let $d=\operatorname{deg} f$. As in [P, §3],

$$
\begin{gathered}
2 g^{\prime}-2=d(2 g-2)+\sum d\left(\alpha_{i}-1\right) / \alpha_{i}, \\
b^{\prime}=d b-\sum d \beta_{i} / \alpha_{i} .
\end{gathered}
$$


But by $(2.8 .3),-P_{X} \cdot P_{X}=(1 / d)\left(-P_{Y} \cdot P_{Y}\right)$; plugging in yields the result.

Remark 3.3. As W. Neumann points out in [N2, p. 251], for $(X, o)$ quasihomogeneous, the expression on the right of (3.2.1) also computes $\chi^{2} /|e|$, where $\chi$ and $e$ arise from the Poincare series of the graded ring. In fact, $\chi$ and $e$ also have interpretations as the euler characteristic and euler number of the Seifert fibration $M \rightarrow C$. Further, Neumann shows that for non-log-canonicals, there is a natural geometry on the link, plus a (naturally normalized) metric, for which the volume of the link is $4 \pi^{2} \chi^{2} /|e|$. That is why the expression on the right of (3.2.1) is a characteristic number. Since every singularity link can be decomposed into Seifert manifolds, it would be interesting to interpret the $-P \cdot P$ invariant in the general case from the Seifert case. We summarize this result:

Corollary 3.4. Let $(X, O)$ be an isolated quasi-homogeneous normal surface singularity which is not log-canonical. Then there is a natural metric on the link so that the volume is $4 \pi^{2}(-P \cdot P)$.

Corollary 3.5. Let $(X, o)$ be a quasi-homogeneous Gorenstein surface singularity, which is not log-canonical. Then $-P \cdot P \geq 1 / 42$, with equality only for the triangle singularity $x^{2}+y^{3}+z^{7}=0$.

Proof. We offer two proofs. First, the Gorenstein property implies that there is an integer $t$ satisfying

$$
\begin{gathered}
t \beta_{i} \equiv 1 \bmod \alpha_{i}, \quad \text { all } i, \\
2 g-2=t b-\sum\left\{b \beta_{i} / \alpha_{i}\right\},
\end{gathered}
$$

where $\{\alpha\}$ means least integer $\geq \alpha$ (e.g., [W2, (4.4.2)]). These imply that

$$
t\left(b-\sum \beta_{i} / \alpha_{i}\right) \leq 2 g-2+r-\sum\left(1 / \alpha_{i}\right) .
$$

Denote the right side term of the inequality by $\chi$, and note $\chi>0$ and $t>0$ if the singularity is not log-canonical. In this situation the last inequality gives $-P \cdot P \geq t \chi$, and it is easy to see that $\chi \geq 1 / 42$.

A second proof follows a suggestion of Robert Bryant. Let $M$ be the unit tangent bundle of a compact Riemann surface $C$ of genus $g \geq 2$. Using the natural metric, the volume of $M$ is (using Gauss-Bonnet) equal to $2 \pi \chi(C) \cdot 2 \pi=$ $8 \pi^{2}(g-1)$. By [P] or [D], the link $L$ has a Galois $G$-covering by $M^{\prime}$, which is an unramified cyclic covering of $M$; in fact, $M^{\prime}$ is the unit sphere bundle of some appropriate $n$th root of the tangent bundle (actually, $n=t$ above). $G$ acts faithfully on $C$; so by Hurwitz's Theorem, $|G| \leq 84(g-1)$. Thus,

$$
\operatorname{Vol}(L)=\operatorname{Vol}\left(M^{\prime}\right) /|G|=n \operatorname{Vol}(M) /|G|=8 \pi^{2}(g-1) n /|G| \geq 4 \pi^{2} n / 42 \text {. }
$$

Comparing with Corollary 3.4 gives the result.

(3.6) We point out finally that $-P \cdot P$ is easily computed from the degrees and weights of a weighted homogeneous complete intersection. For instance, let 
$f\left(z_{0}, z_{1}, z_{2}\right)$ be a weighted homogeneous polynomial, of weights $w_{0}, w_{1}, w_{2} \in$ $\mathbb{Q}$; thus, letting $z_{i}$ have weight $w_{i}, f$ has degree 1 .

Proposition 3.7 (cf. [Wt, 1.15]). If $(X, o)$ is a weighted homogeneous hypersurface singularity, not a rational double point, then $-P \cdot P=\left(1-\sum w_{i}\right)^{2} / \Pi w_{i}$.

\section{BIBLIOGRAPHY}

[D] I. Dolgachev, On the link space of a Gorenstein quasihomogeneous surface singularity, Math. Ann. 265 (1983), 529-540.

[G] M. Gromov, Volume and bounded cohomology, Inst. Hautes Études Sci. Publ. Math. 56 (1982), 5-99.

[K] Y. Kawamata, Crepant blowing-up of 3-dimensional canonical singularities and its application to degenerations of surfaces, Ann. of Math. 127 (1988), 93-163.

[L] H. Laufer, Weak simultaneous resolution for deformations of Gorenstein surface singularities, Proc. Sympos. Pure Math., vol. 40, part 2, Amer. Math. Soc., Providence, RI, 1983, pp. 1-29.

[MT] J. Milnor and W. Thurston, Characteristic numbers of 3-manifolds, Enseign. Math. 23 (1977), 249-254.

[Mo] M. Morales, Calcul de quelques invariants des singularités de surface normale, Enseign. Math. 31 (1983), 191-203.

[N1] W. Neumann, A calculus for plumbing applied to the topology of complex surface singularities and degenerating complex curves, Trans. Amer. Math. Soc. 286 (1981), 299-344.

[N2] _ Geometry of quasihomogeneous surface singularities, Proc. Sympos. Pure Math., vol. 40, part 2, Amer. Math. Soc. Providence, RI, pp. 245-258.

[P] H. Pinkham, Normal surface singularities with $\mathbb{C}^{*}$-action, Math. Ann. 227 (1977), $183-193$.

[S] F. Sakai, Anticanonical models of rational surfaces, Math. Ann. 269 (1984), 389-410.

[Wg] P. Wagreich, Singularities of complex surfaces with solvable local fundamental group, Topology 11 (1972), 51-72.

[W1] J. Wahl, Vanishing theorems for resolutions of surface singularities, Invent Math. 31 (1975), 17-41.

[W2] _- Deformations of quasi-homogeneous surface singularities, Math. Ann. 280 (1988), 105-128.

[W3] _ Miyaoka's inequality for a normal surface, in preparation.

[Wt] K. Watanabe, On plurigenera of normal isolated singularities. I, Math. Ann. 250 (1980), 65-94.

Department of Mathematics, University of North Carolina, Chapel Hill, North CAROLINA 27599-3250 